\title{
An SNR-based admission control scheme in WiFi- based vehicular networks
}

\author{
Kihun Kim ${ }^{1}$, Younghyun Kim ${ }^{1}$, Sangheon Pack ${ }^{1 *}$ and Nakjung Choi ${ }^{2}$
}

\begin{abstract}
In WiFi-based vehicular networks, the performance anomaly problem can be serious because different vehicles with diverse channel conditions access the channel by a random access protocol. In this article, we first develop a novel analytical model, which combines the vehicular traffic theory and WiFi properties to show the impact of performance anomaly at the intersection. We then propose a signal-to-noise ratio (SNR)-based admission control scheme that excludes vehicles with bad channel qualities to address the performance anomaly problem. Extensive simulation and analytical results are presented to show the effect of the traffic condition and the topology. From the simulation results, it can be found that the SNR-based admission control scheme can improve the overall throughput, and starvation issues can be addressed by means of mobility in WiFi-based vehicular networks with multiple intersections.
\end{abstract}

Keywords: performance anomaly, WiFi-based vehicular networks, SNR-based admission control, mobility

\section{Introduction}

Recently significant progress has been made in vehicular networks to support mobile users. Vehicular communications can be classified into vehicle-to-infrastructure (V2I) and vehicle-to-vehicle (V2V) communications. In terms of communication technology, wireless local area network (WLAN) or WiFi [1], wireless wide area network (WWAN) [2], or their combination [3] can be used in vehicular environments. Even though the performance of WWAN has been improved over the past years, its data rate is still limited compared with WLAN. Also, WWAN typically adopts a meter-rate-dependent monetary cost policy which is a burden to users. Meanwhile open WiFi networks are deployed in many cities around the world and thus WiFi-based vehicular networks are perceived as one of the most promising solutions.

In WiFi-based vehicular networks, users traveling by car usually come in range of multiple WiFi access points (APs). While they are on their way, mobile users experience intermittent connectivity because of the short

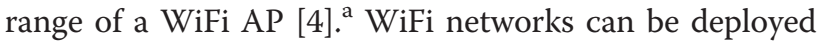
at road sides and intersections. When vehicles are in

\footnotetext{
* Correspondence: shpack@korea.ac.kr

${ }^{1}$ School of Electrical Engineering, Korea University, Seoul, Korea

Full list of author information is available at the end of the article
}

WiFi networks deployed at intersections, more dynamic channel qualities can be observed compared with vehicles on the road side. This is because there always exist stopped cars as well as moving cars at intersections. It is known that stopped cars have better channel qualities than moving cars [5], and vehicles also experience different channel conditions dependent on the distance from an AP. In addition, stopped cars at intersections have much longer association time with WiFi APs than moving cars at road sides and thus the association time at an intersection needs to be used efficiently.

In carrier sensing multiple access/collision avoidancebased WLANs, each node has the same opportunity to access the channel, and the channel utilization by a node can be defined as the ratio between the transmission time of the node and the total transmission time of all other nodes. Then, nodes transmitting at high transmission rates obtain the same throughput as the nodes transmitting at low transmission rates, which is known as performance anomaly [6]. Because vehicles at an intersection have various channel conditions, throughput degradation because of performance anomaly happens more apparently at intersections rather than the road sides. To solve the performance anomaly problem, several studies have been proposed, in which both fast and slow nodes capture the channel for the same amount of 
time by means of packet fragmentation [7], backoff adaptation [8], or packet aggregation [9]. However, these studies do not investigate the effect of mobility on the performance anomaly problem.

In this article, we investigate the performance anomaly problem at the intersection where stopped and moving cars exist. In particular, we develop a novel analytical model, which combines the vehicular traffic theory and WiFi properties to show the impact of performance anomaly at the intersection. We also propose a signalto-noise ratio (SNR)-based admission control scheme that excludes vehicles with bad channel qualities as a remedy for the performance anomaly problem. Extensive simulation and analytical results are presented to show the effect of the traffic condition and the topology, which demonstrate that the SNR-based admission control scheme can improve the overall throughput, and starvation issues can be addressed by means of mobility in WiFi-based vehicular networks with multiple intersections. To the best of the authors' knowledge, this is the first study to investigate and solve the performance anomaly problem in WiFi-based vehicular networks.

The rest of this article is organized as follows. In Section 2, related studies are summarized and the system model is given in Section 3. We then analyze the performance anomaly problem at the intersection in Section 4 and present an SNR-based admission control scheme in Section 5. Simulation and analytical results are given in Section 6. Finally, Section 7 concludes this article with future studies.

\section{Related study}

Ott and Kutscher [4] introduced the idea of Drive-thru Internet systems where the connection between a vehicle and a roadside AP is opportunistically established during a travel, and they studied the performance of UDP and TCP flows in vehicles moving at different speeds. Subsequently, many studies have been conducted to verify the feasibility of WiFi-based vehicular networks [10] and to improve the performance of Internet access in moving vehicles [11]. Also, interesting studies have been done to develop analytic models that characterize salient features of Drive-thru systems [12]. However, most of them assume low traffic density (i.e., a single vehicle in the coverage of an AP).

In urban environments, because of high traffic density, the channel contention among vehicles should be considered. In a recent study [13], an analytical model is presented by considering the channel contention. However, this study does not consider the performance anomaly problem. In WiFi-based vehicular networks, vehicles in the coverage of an AP have different transmission rates because the channel quality degrades proportionally to the distance from an AP and varies depending on the velocity of vehicles. Hence, we need to consider not only the channel condition but also the diversity of transmission rates in urban environments. In this study, we analyze the communication performance of a vehicle by considering the performance anomaly problem that occurs in multi-rate environments.

To solve the performance anomaly problem, a number of schemes have been presented in the literature. Most of them allow both nodes with high and low transmission rates to capture the channel for the same amount of time, i.e., the time fairness is sustained. By doing so, the throughput degradation of nodes with high transmission rates can be mitigated. The existing schemes can be classified into three categories: packet fragmentation [7], contention window adaptation [8], and packet aggregation [9]. However, all of these studies do not consider mobility. On the contrary, we try to solve the performance anomaly problem by adopting admission control with the help of mobility in vehicular environments.

\section{System model}

In this study, we adopt a macroscopic vehicle model that aggregates vehicles into a flow and describes the flow in terms of speed, density, and vehicle arrival rate. At an intersection, there exist many traffic flows depending on the number of road segments and these flows can be classified into two types: green light flow and red light flow. A green light flow is an aggregation of vehicles allowed to proceed with the green light signal whereas a red light flow is a set of stopped vehicles at each road segment. These two types of flows are separately investigated in this section. We summarized notations and their meanings in Table 1.

We first consider the green light flow (i.e., moving vehicles) as shown in Figure 1. $S$ is the length of space for each vehicle. $L_{i}$ is the transmission range of an AP when the specific transmission rate $r_{i}$ is used. $L_{i}$ is less than or equal to the maximum transmission range of an AP. Let $q$ be the vehicle arrival rate that counts the number of vehicles passing a fixed roadside observation point per unit time. $v$ is the vehicle speed and $k$ is the vehicle density. Then, we have the following relationship:

$$
q=k v .
$$

Based on [14], it can be found that there exists a linear relationship between the speed $v$ and the density $k$ as

$$
v=v_{f}\left(1-\frac{k}{k_{\text {jam }}}\right)
$$

where $v_{f}$ is the free-flow speed (i.e., the speed when the vehicle is alone on the road) and $k_{\text {jam }}$ is the traffic 


\section{Table 1 Notations and meanings}

\begin{tabular}{|c|c|}
\hline Notation & Meaning \\
\hline 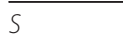 & Length of space for each vehicle \\
\hline$L_{i}$ & $\begin{array}{l}\text { Transmission range of an AP when the specific transmission } \\
\text { rate } r_{i} \text { is used }\end{array}$ \\
\hline 9 & $\begin{array}{l}\text { Vehicle arrival rate that counts the number of vehicles } \\
\text { passing a fixed roadside observation point per unit time }\end{array}$ \\
\hline$v$ & Vehicle speed \\
\hline k & Vehicle density \\
\hline$v_{f}$ & Speed when the vehicle is alone on the road \\
\hline$k_{\text {jam }}$ & Traffic jam density when traffic flow comes to a halt \\
\hline$\lambda$ & Mean vehicle arrival rate \\
\hline$T_{i}$ & Sojourn time in the coverage of $2 L_{i}$ \\
\hline$p_{N}$ & $\begin{array}{l}\text { Steady state probability with } N \text { vehicles in the coverage of } \\
\text { an AP }\end{array}$ \\
\hline$C_{i}$ & Maximum number of vehicles in the coverage of an AP \\
\hline I & Elapsed time since the light turned red \\
\hline M & $\begin{array}{l}\text { Number of remaining vehicles after the last green light } \\
\text { signal }\end{array}$ \\
\hline E & Number of outgoing vehicles during the green light signal \\
\hline$T(N)$ & $\begin{array}{l}\text { Overall transmission time of a single node when } N \text { nodes } \\
\text { exist }\end{array}$ \\
\hline$t_{\mathrm{tr}}$ & MAC protocol data unit (MPDU) transmission time \\
\hline$t_{\text {ack }}$ & MAC acknowledgement transmission time \\
\hline$t_{\mathrm{pr}}$ & $\begin{array}{l}\text { Physical layer convergence protocol (PLCP) preamble and } \\
\text { header transmission time }\end{array}$ \\
\hline$t_{\mathrm{ov}}$ & $\begin{array}{l}\text { Constant overhead that includes DIFS }=50 \mu \mathrm{s}, \mathrm{SIFS}=10 \mu \mathrm{s} \text {, } \\
t_{\mathrm{ack}} \text {, and } t_{\mathrm{pr}}\end{array}$ \\
\hline$t_{\text {cont }}(N)$ & Channel contention time when $N$ nodes exist \\
\hline$G_{i}$ & Group of nodes with the ith highest transmission rate $r_{i}$ \\
\hline$N_{i}$ & Number of nodes included in $G_{i}$ \\
\hline$t_{\mathrm{ov}}^{r_{i}}$ & Constant overhead time of a node in $G_{i}$ \\
\hline$T_{i}$ & Overall transmission time of a node in $G_{i}$ \\
\hline$T_{\text {jam }}(N)$ & Average time spent by collisions when $N$ nodes exist \\
\hline$b$ & Number of groups \\
\hline$P_{C}(N)$ & Conditional collision probability when $N$ nodes exist \\
\hline$C W_{\min }$ & Minimum contention widow size \\
\hline$X_{1}$ & Expected throughput of a super group $(1, \ldots, l)$ \\
\hline $\mathrm{SNR}_{i}$ & SNR threshold to be a member of $G_{i}$ \\
\hline
\end{tabular}

jam density when traffic flow comes to a halt that is given by $k_{\text {jam }}=1 / S$.

Given the vehicle arrival rate, $v$ and $k$ can be determined by using Equations 1 and 2 which describe a homogeneous and equilibrium traffic flow passing through a road segment [15]. From [16], the vehicle arrival can be approximated as a Poisson process with mean rate $\lambda$ (i.e., $q=\lambda$ ). It is assumed that every vehicle has the same speed $v$ and the constant sojourn time $T_{i}$ in the coverage of $2 L_{i}$. Therefore, the sojourn time $T_{i}$ can be obtained as $T_{i}=2 L_{i} / v$. Under these conditions, an $M / D /$ $C / C$ queueing system can be used to model the green light flow [13]. Then, the steady-state probability $p_{N}$ with $N$ vehicles in the coverage of an AP $2 L_{i}$ is given by

$$
p_{N}=\frac{\left(\lambda T_{i}\right)^{N} / N !}{\sum_{j=0}^{C_{i}}\left(\lambda T_{i}\right)^{j} / j !}
$$

where $0 \leq N \leq C_{i}$. $C_{i}$ is the maximum number of vehicles in the coverage of an AP, which is given by $C_{i}=$ $2 k_{\text {jam }} L_{i}=2 L_{i} / S$. Based on this model, when the vehicle arrival rate $q$ is given, the number of vehicles using a specific transmission rate can be computed.

As shown in Figure 2, in a red light flow, vehicles are accumulated until the end of the red light signal. Hence, the elapsed time since the light turned red $I$ is an important factor to model the red light flow. $M$ is the number of remaining vehicles after the last green light signal. During $I$, there will be incoming vehicles with rate $q$ and thus the number of arrived vehicles is $q I$. Therefore, the number of remaining vehicles, $M$, is given by $q I-E$ where $E$ is the number of outgoing vehicles during the green light signal and $E$ can be estimated from simulation results. On the other hand, if the number of outgoing vehicles is larger than that of incoming vehicles, $M$ is simple 0 . Consequently, $M$ can be expressed as

$$
M= \begin{cases}q I-E, & \text { for } q I \geq E \\ 0, & \text { otherwise }\end{cases}
$$

As shown in Figure 3, the number of vehicles in the transmission range $L_{i}$ increases up to $L_{i} / S$ with the increase of $I$ and remains at the value. By taking an integral of the area in Figure 3 for given input rate $q$ and time period $I$, the average number of vehicles during the time period $I$ in the range of $L_{i}$ can be obtained as

$$
E[N]=\frac{1}{I} \int_{0}^{I} \min \left(M+q t, L_{i} / S\right) d t .
$$

In short, if the number of road segments at an intersection and the arrival rate of vehicles at each road segment are known, the number of vehicles using specific transmission rates can be found from Equations 3 and 5 .

\section{Problem statement}

When $N$ nodes exist in an IEEE 802.11 WLAN, the overall transmission time $T(N)$ can be expressed as

$$
T(N)=t_{\mathrm{tr}}+t_{\mathrm{ov}}+t_{\mathrm{cont}}(N)
$$

where $t_{\mathrm{tr}}$ is the MAC protocol data unit (MPDU) transmission time and $t_{\mathrm{ov}}$ is the constant overhead that includes DIFS $=50 \mu \mathrm{s}$, SIFS $=10 \mu \mathrm{s}$, MAC acknowledgement transmission time $t_{\text {ack }}$, and physical layer convergence protocol (PLCP) preamble and header transmission time $t_{\mathrm{pr}} \cdot t_{\mathrm{pr}}$ is dependent on the selected transmission rate. For example, when the transmission 


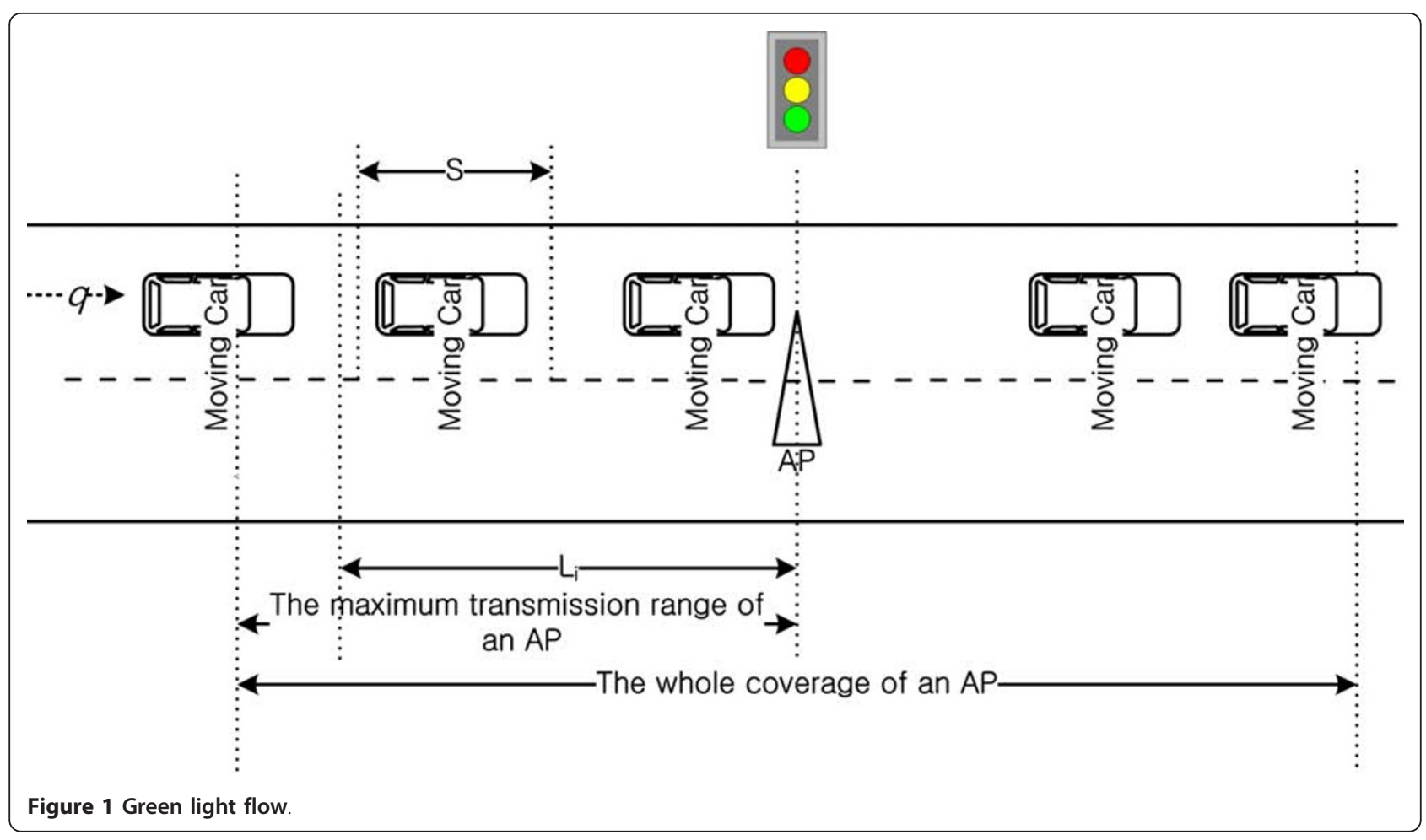

rate is $1 \mathrm{Mbps}, t_{\mathrm{pr}}$ is $192 \mu \mathrm{s}$ and $t_{\mathrm{pr}}$ is $96 \mu \mathrm{s}$ for other transmission rates. $t_{\text {cont }}(N)$ is the time due to channel contention.

Assume that $N$ nodes use different transmission rates and they can be classified into several groups depending on the transmission rate. Let $G_{i}$ be the group of nodes with the $i$ th highest transmission rate $r_{i}$ and $N_{i}$ be the number of nodes included in $G_{i} \cdot t_{\mathrm{ov}}^{r_{i}}$ is the constant overhead of a node in $G_{i}$. If the propagation time is neglected, the overall transmission time of a node in $G_{i}$

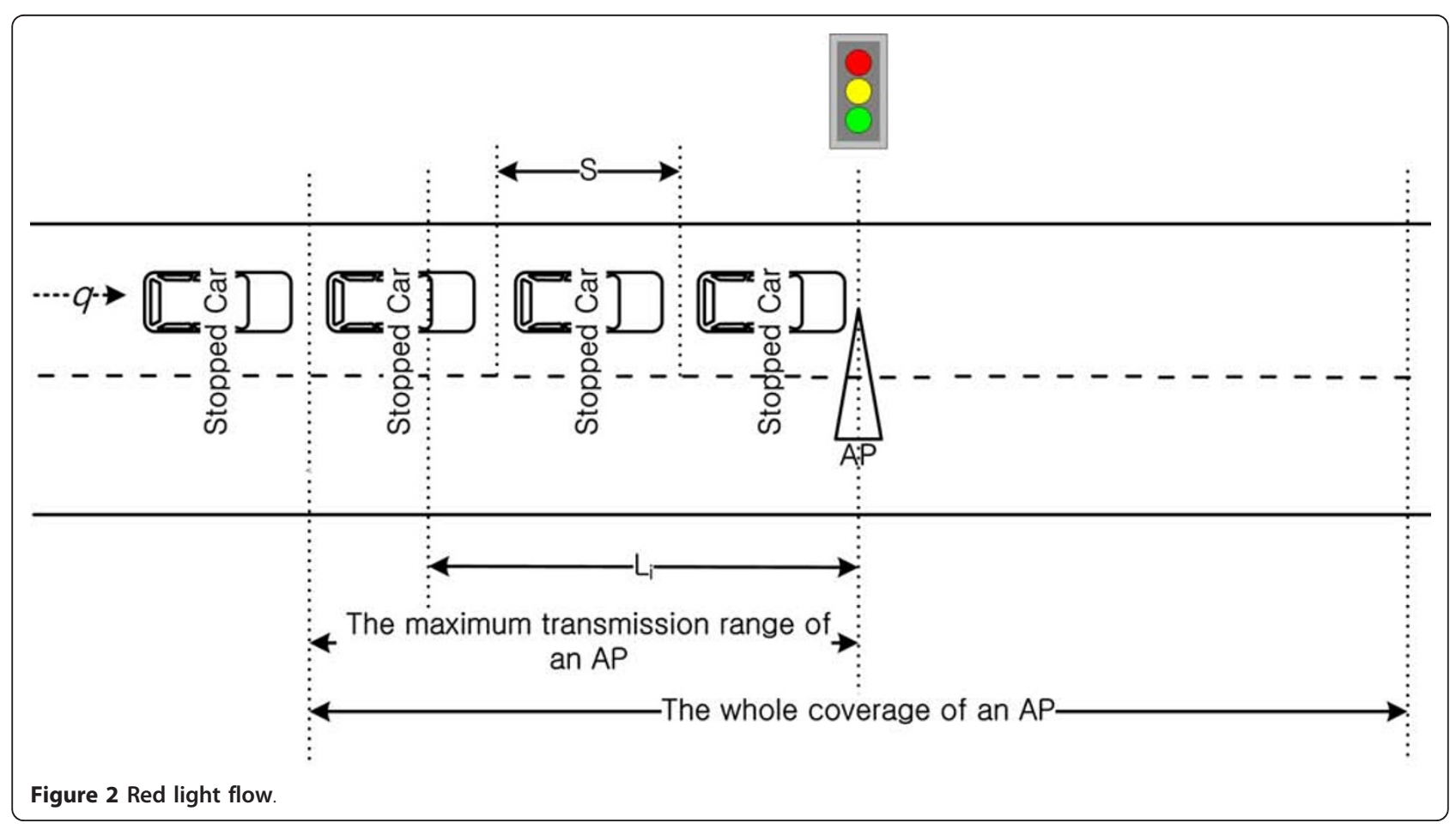




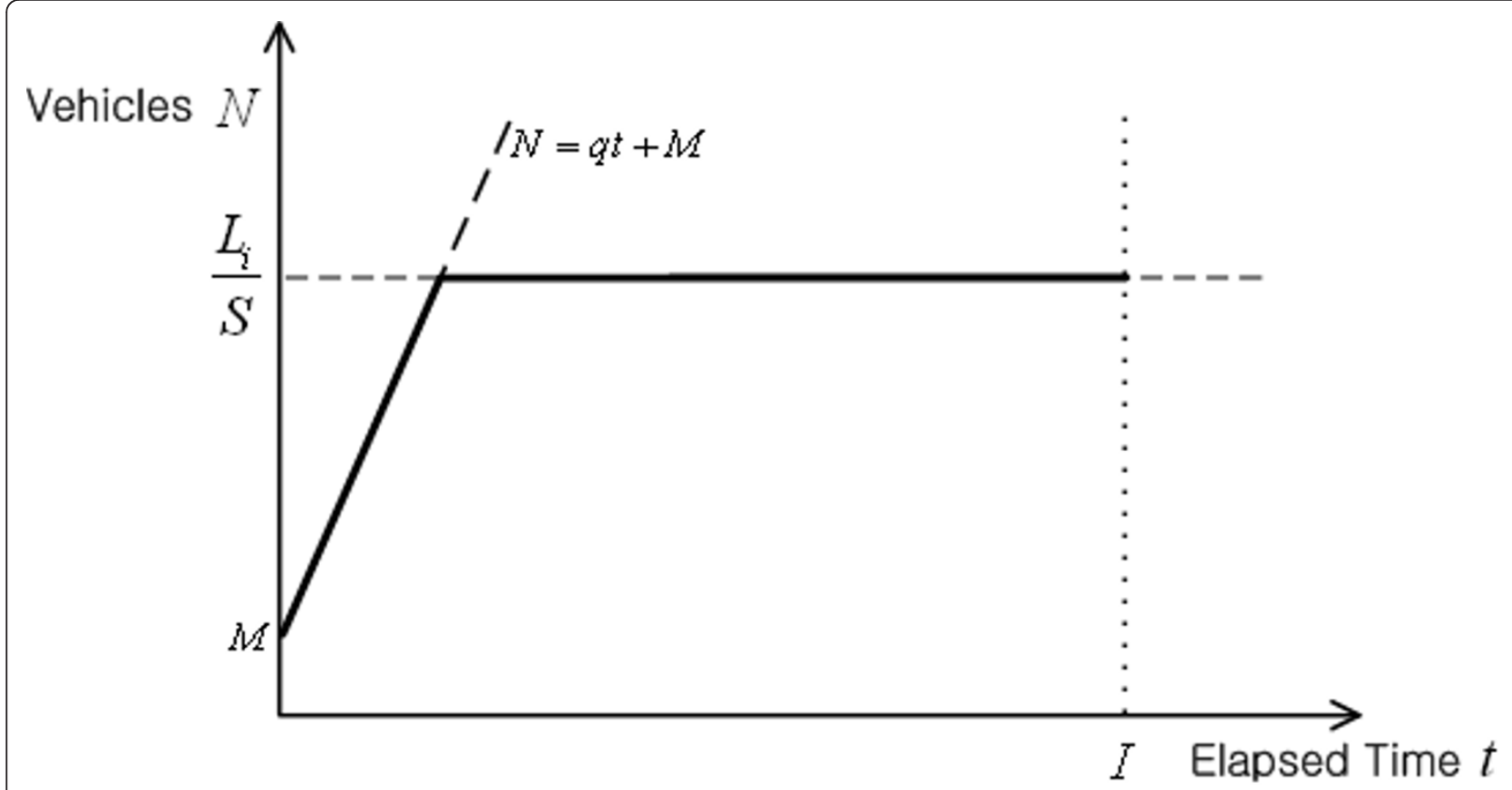

Figure 3 Number of vehicles in AP transmission range versus elapsed time.

is

$$
T_{i}=\frac{D}{r_{i}}+t_{\mathrm{ov}}^{r_{i}}+t_{\text {cont }}(\mathrm{N})
$$

where $D$ is the MPDU size.

Based on [6], it can be found that each node has the same throughput $X$ regardless of transmission rates, and $X$ is obtained from

$$
X=\frac{D}{\sum_{i=1}^{b} N_{i} \times T_{i}+P_{C}(N) \times T_{\mathrm{jam}}(N) \times N}
$$

where $T_{\text {jam }}(N)$ is the average time spent by collisions and $b$ is the number of groups. $P_{C}(N)$ is the conditional collision probability that is given by

$$
P_{C}(N)=1-\left(1-\frac{1}{C W_{\min }}\right)^{N-1}
$$

where $C W_{\min }$ is the minimum contention widow size.

From Equation 8, it can be seen that the throughput is determined by the number of nodes and the transmission time. Since a node with a low transmission rate leads to longer transmission time, the throughput $X$ will be decreased significantly as the number of nodes with lower transmission rates increases, which is a wellknown performance anomaly problem [6]. Figure 4 shows the throughput of a node when only one node uses $11 \mathrm{Mbps}$ and others use lower rates $(1,2$, or 5.5 Mbps). Owing to the performance anomaly, the throughput decreases as the number of nodes using low rates increases. Apparently, the throughput drop happens more seriously when $1 \mathrm{Mbps}$ nodes exist compared with the case when 2 or 5.5 Mbps nodes exist.

As mentioned earlier, two types of cars exist at an intersection: stopped and moving cars (see Figure 5). Typically, moving cars may have worse channel quality (i.e., lower transmission rate) than stopped cars [5]. Moreover, stopped cars have different transmission rates because the channel quality degrades proportionally to the distance from the AP. Consequently, the performance anomaly problem is more serious at intersections in vehicular networks. To mitigate the impact of performance anomaly, we introduce an SNR-based admission control scheme in Section 5, which limits the number of nodes with low transmission rates for improving the overall throughput.

\section{SNR-based admission control scheme}

Most of the schemes to address the performance anomaly problem guarantee the time fairness among nodes regardless of their transmission rates. Our approach is different from them because the proposed scheme does not allow nodes with low transmission rates to capture the channel at all to prevent the performance anomaly problem via admission control. The amount of time taken from nodes with low transmission rates can be used by nodes with high transmission rates, and thus high rate nodes can transmit more packets than low rate nodes during the same time period. Consequently, 


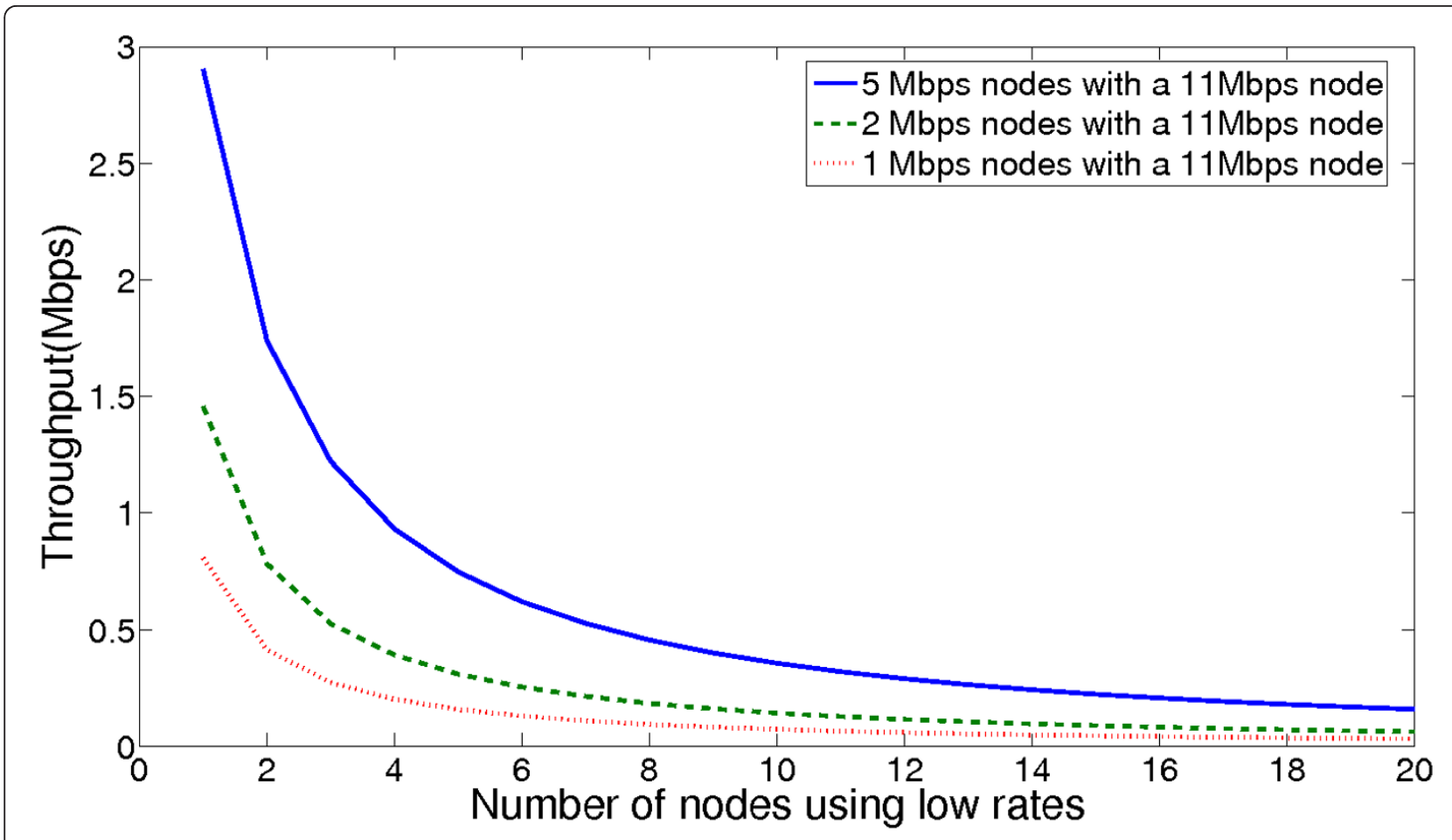

Figure 4 Throughput of an IEEE 802.11b node when only one node uses $11 \mathrm{Mbps}$ and others use lower rates.

the overall performance of IEEE 802.11 networks can be improved.

Specifically, we propose an SNR-based admission control scheme where the AP estimates the SNR through association procedures. ${ }^{\text {b }}$ IEEE 802.11 WLAN defines two scanning modes for association: active and passive scannings. In both modes, a vehicle can estimate the SNR by receiving a probe response (in active mode) or beacon frames (in passive mode). After that, the estimated SNR information is reported to the AP by means of an association request frame. ${ }^{\mathrm{c}}$ Based on the SNR estimation, the AP performs an admission control scheme which key idea is to exclude vehicles with low transmission rates. By doing so, the performance anomaly problem can be mitigated and the overall throughput can be improved. The detailed procedure is as follows.

1) All the vehicles in the range of the AP estimate their SNRs after receiving a beacon frame or a probe response frame from the AP. To mitigate the effect of SNR variations, SNR values from multiple beacon or probe response frames can be averaged [17].

2) An association request frame including the estimated SNR is sent to the AP.

3) The AP classifies vehicles into $b$ groups from $G_{1}$ to $G_{b}$ based on the transmission rate selected depending on the SNR.
4) As shown in Table 2, the AP constructs a decision criterion table that includes the expected throughput of a super group $(1, \ldots, l)$, which is a union of groups from $G_{1}$ to $G_{l}$, is given by

$X_{l}=\frac{D}{\sum_{i=1}^{l} N_{i} \times T_{i}+P_{C}(N) \times T_{\mathrm{jam}}(N) \times N}$

where $1 \leq l \leq b$. This table also includes member groups of the super group, the proportion of vehicles involved in the super group, and $\mathrm{SNR}_{i}$ which is the SNR threshold to be a member of $G_{i}$.

5) From Table 2, the AP can check the threshold SNR to keep the expected throughput above a certain value. For example, $\mathrm{SNR}_{1}$ can be selected to provide vehicles with throughput higher than $X_{2}$. Note that $\mathrm{SNR}_{x}>\mathrm{SNR}_{y}$ and $X_{x}>X_{y}$ if $x<y$ where $x, y \in\{1, \ldots, b\}$. This is because the overall throughput degrades when vehicles with low transmission rates are associated with an AP (see Section 4).

6) Finally, the AP sends an association response frame only to vehicles with higher SNRs than the threshold.

Figures 6 and 7 show detailed actions performed by 


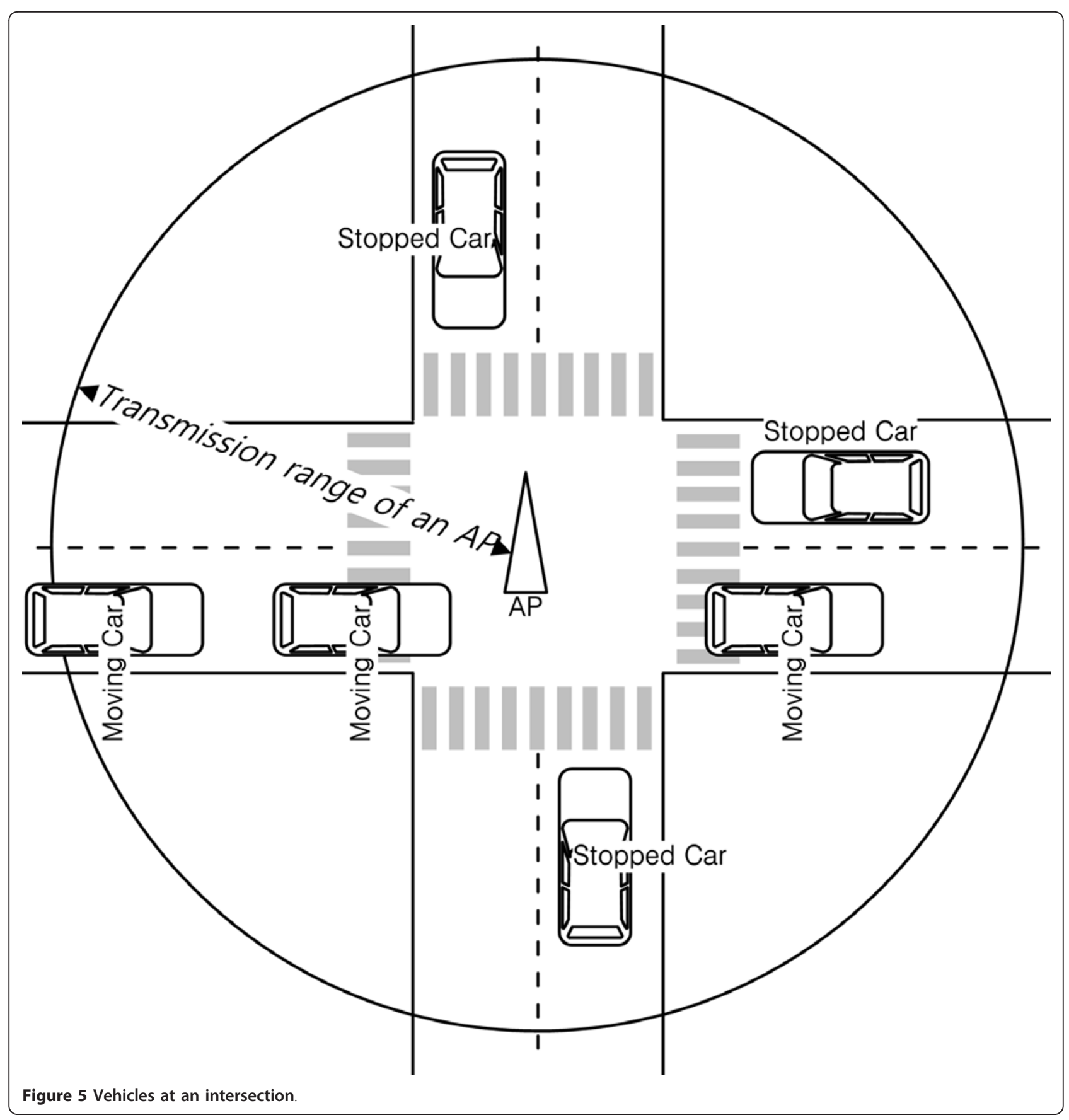

the AP and the vehicle, respectively. Note that the proposed admission control scheme can be implemented at the commodity WiFi standards without any significant modifications.

In the SNR-based admission control scheme, vehicles associated with an AP can communicate without any significant impact of performance anomaly. However, other vehicles excluded through admission control cannot transmit data at all. Consequently, the SNR-based admission control scheme may lead to a starvation issue at an AP. However, when we consider mobility over a traveling path with a number of APs, the starvation problem can be addressed as follows. In urban environments, a vehicle usually passes through multiple intersections during a travel and opportunistically experiences various channel conditions. At an intersection, the vehicle may be excluded as a result of admission control and thus may not receive any data. However, at other intersections after movements, the vehicle can communicate with APs, i.e., after moving 
Table 2 Decision criterion table

\begin{tabular}{llll}
\hline Throughput & Groups & Proportion of groups & Threshold SNR \\
\hline$X_{1}$ & $G_{1}$ & $N_{1} / N$ & $\mathrm{SNR}_{1}$ \\
$X_{2}$ & $G_{1}+G_{2}$ & $N_{1}+N_{2} / N$ & $\mathrm{SNR}_{2}$ \\
$\ldots$ & $\sum^{b}$ & $\ldots$ & $\ldots$ \\
$X_{b}$ & $\sum_{i=1} G_{i}$ & 1 & $\mathrm{SNR}_{b}$ \\
\end{tabular}

entire traveling path the vehicle can receive more data than the case in which admission control is not used. More investigations on the starvation issue will be given in the following section through simulations.

\section{Performance evaluation}

In this section, we evaluate the performance of the SNR-based admission control scheme and investigate the effect of performance anomaly at an intersection via VISSIM [18] and analysis. VISSIM is a micro-simulation software developed to model urban traffic environments. In VISSIM, car movement models are categorized into Wiedemann 74 and Wiedemann 99 models. The Wiedemann 74 model is suitable in urban environments, whereas the Wiedemann 99 model is applicable to inter-urban environments [19]. We choose the Wiedemann 74 model for simulating urban traffic environments. Unless explicitly mentioned, the network topology consists of three-lane roads and four-way intersections with IEEE 802.11b WLAN APs. At a multi-way intersection, a traffic signal cycle consists of red and green light signals and it is assumed only one lane is allowed to pass at the same time. The AP transmission ranges are dependent on transmission rates as shown in Table 3[20]. Other WLAN parameters follow the IEEE $802.11 \mathrm{~b}$ standard values and the frame size is 1500 bytes. In an intersection, we use a fixed time traffic control system that operates with the constant cycle length, phase sequence, and green time during every cycle. In this study, we focus on elastic data download services in vehicular network with opportunistic (or intermittent) WiFi connectivity, and thus the handoff and car start times are not considered. Therefore, the throughput can be computed by dividing the total amount of data during travel with the total traveling time.

\section{A. Effect of traffic condition}

We first evaluate the network throughput at a single intersection for each super group with respect to the vehicle arrival rate. First of all, Figure 8 indicates that the simulation results are well matched with the analytical results except the super group $(1,2,3,4)$. The analytical model on the number of moving vehicles is based on the $M / D / C / C$ queueing model described in Section

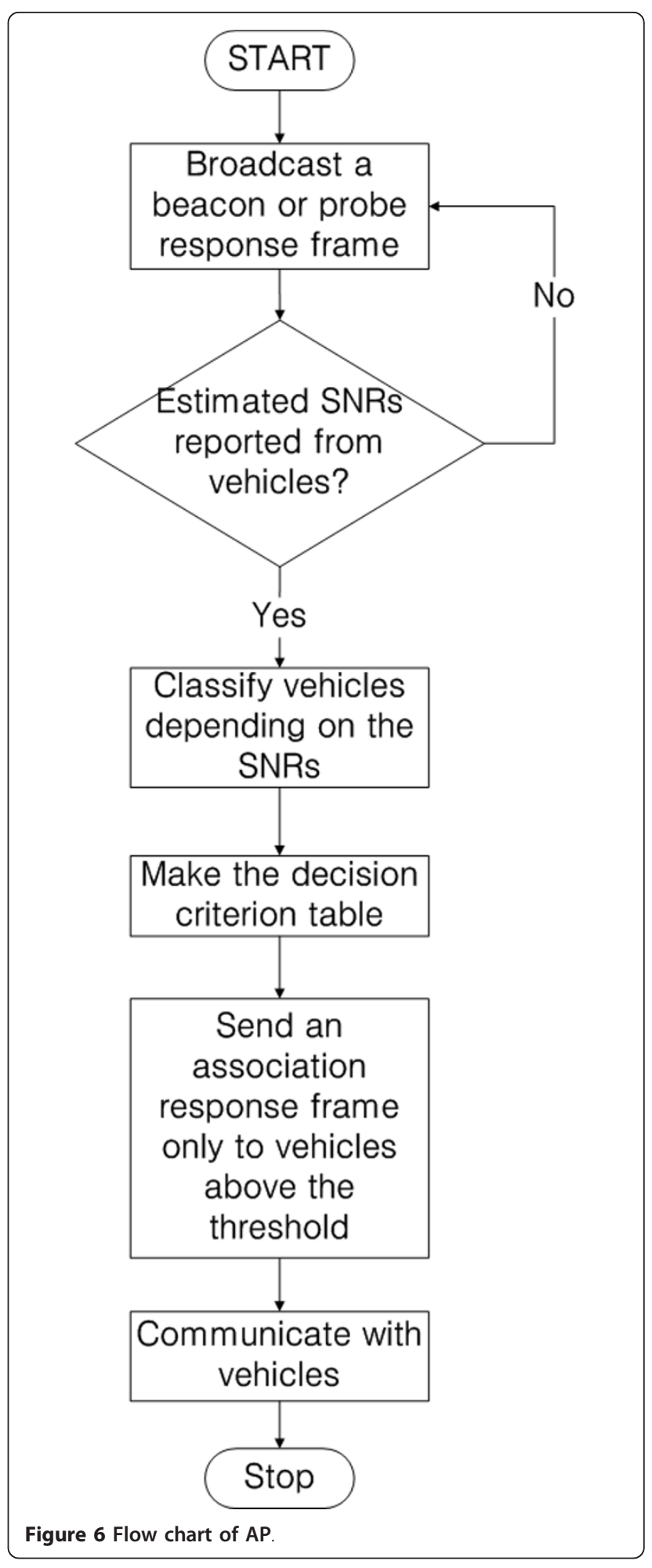

3. It can be found that the analytical numbers are slightly higher than the simulation results, i.e., the analytical result for the super group $(1,2,3,4)$ overestimates the network throughput. 


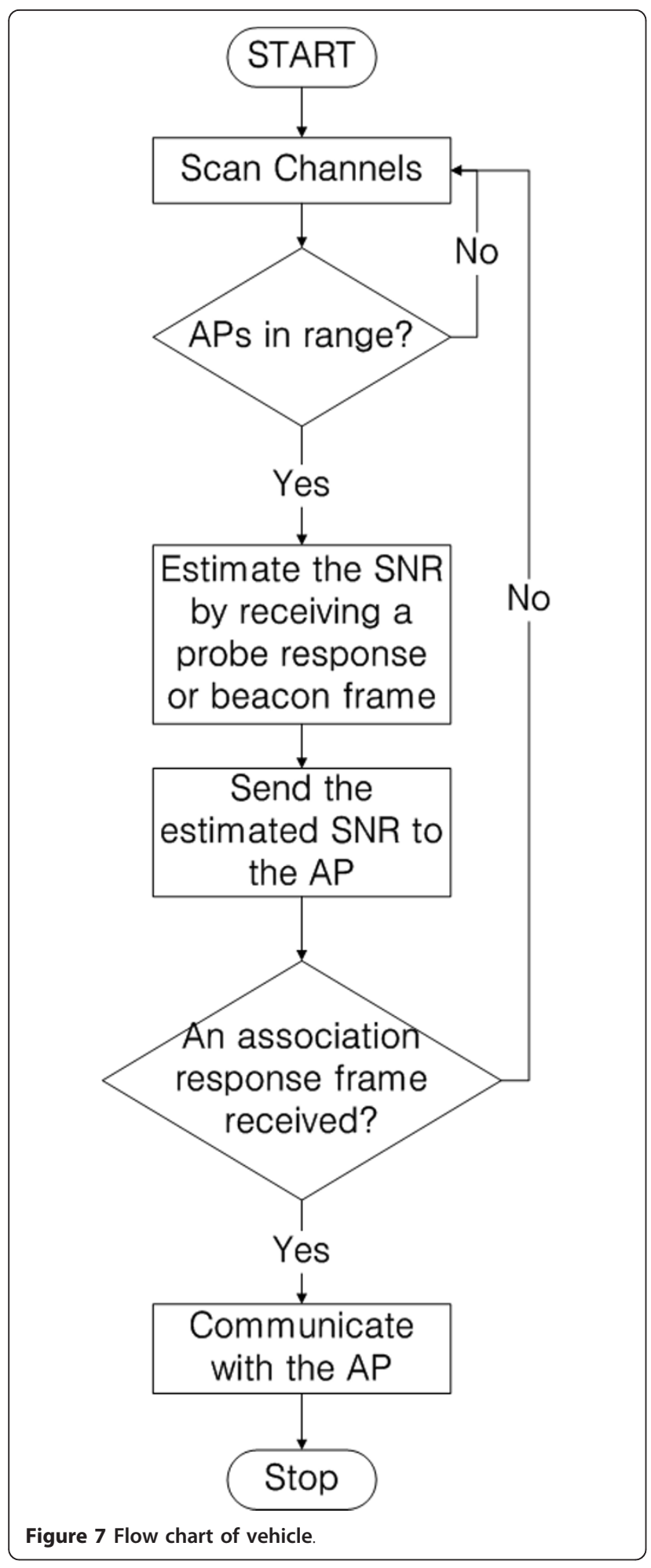

From Figure 8, it can be shown that the network throughput decreases with the increase of the number of vehicles due to more collisions in the IEEE 802.11 MAC layer. Also, Figure 8 indicates that, for a small number of vehicles/hour, the network throughput of
Table 3 Transmission rate vs. transmission range

\begin{tabular}{lllll}
\hline Transmission rate & $\mathbf{1 1} \mathbf{M b p s}$ & $\mathbf{5 . 5} \mathbf{~ M b p s}$ & $\mathbf{2} \mathbf{M b p s}$ & $\mathbf{1} \mathbf{M b p s}$ \\
\hline Range & $48.2 \mathrm{~m}$ & $67.1 \mathrm{~m}$ & $\mathbf{7 4 . 7} \mathrm{m}$ & $100 \mathrm{~m}$ \\
\hline
\end{tabular}

each super group except the super group $(1,2,3,4)$ shows almost the same value. However, when the number of vehicles/hour exceeds 1400 vehicles/hour, the network throughputs of the super groups $(1,2)$ and $(1,2,3)$ drop drastically. This can be explained as follows. When the number of vehicles/hour is less than 1400 vehicles/hour, all these vehicles use $11 \mathrm{Mbps}$ and thus no performance anomaly occurs. On the contrary, if the number of vehicles/hour is larger than 1400 vehicles/hour, the distances between the AP and some vehicles are larger than the $11 \mathrm{Mbps}$ range (i.e., $48.2 \mathrm{~m}$ in Table 3) and therefore these vehicles should use low transmission rates such as 5.5/2/1 Mbps, which results in the throughput degradation due to performance anomaly.

Similarly, it can be shown that the network throughput of the super group $(1,2,3)$ also drops at the point of 1900 vehicles/hour. The network throughput of the super group (1), on the other hand, does not experience significant drops at the points of 1400 and 1900 vehicles/hour. This is because all vehicles associated with the AP can transmit data at the highest transmission rate (i.e., $11 \mathrm{Mbps}$ ) and therefore the performance anomaly problem does not occur. Owing to the same reason, the super group $(1,2)$ does not experience significant throughput degradation at the point of 1700 vehicles/hour.

When no admission control scheme is used (i.e., super group $(1,2,3,4))$, the network throughput is less than a half of the throughputs of the other super groups for every arrival rate because performance anomaly happens in the super group $(1,2,3,4)$ regardless of the arrival rate. From Figure 8 , the network throughput variation remains when the arrival rate exceeds the point of 2500 vehicles/hour. At this point, the transmission range of an AP is already fully occupied with vehicles. Thus, the network throughputs are not affected by the arrival rate any more. Only depending on the groups accepted by the AP, the network throughput is determined.

Figure 9 shows the simulation result on the number of vehicles included in each group under different traffic signal cycle lengths at an one-lane four-way intersection when the vehicle arrival rate is 900 vehicles/hour. Traditionally, the traffic signal cycle length is extended to reduce the start loss time caused by frequent starts in each traffic signal cycle. However, it can be found that as the traffic signal cycle length decreases, the number of stopped vehicles in the transmission range of an AP 


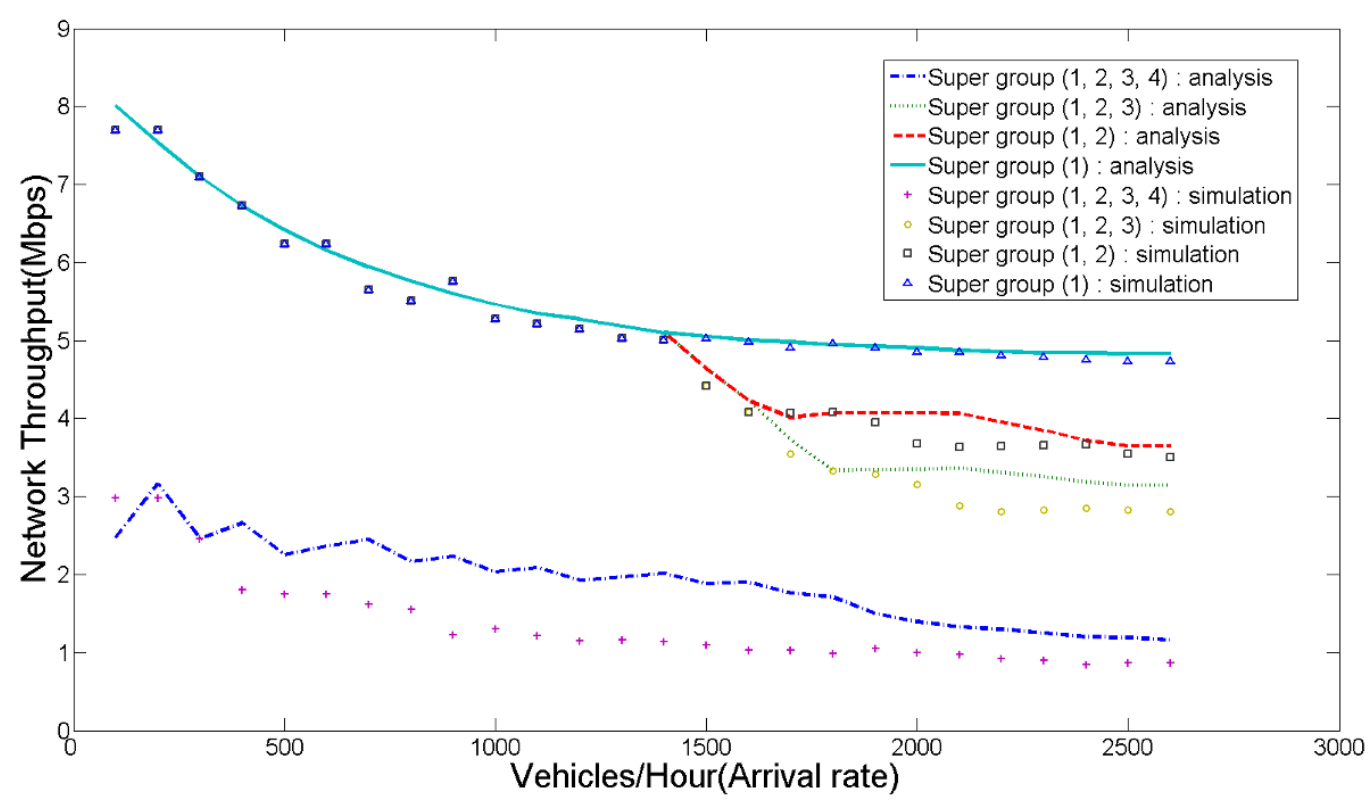

Figure 8 Network throughput versus traffic load (signal cycle length $=140 \mathrm{~s}$ ).

decreases and thus only the group of vehicles with 11 Mbps remains. This is because vehicles are accumulated during a shorter time period as the traffic signal cycle length decreases. As a result, the performance anomaly problem does not happen when the traffic signal cycle length is short.

\section{B. Effect of topology}

In this section, we present simulation results to assess the performance of the proposed admission control scheme under different network topologies.

As demonstrated in Figure 8, even though the super group (1) exhibits the highest throughput, all vehicles in

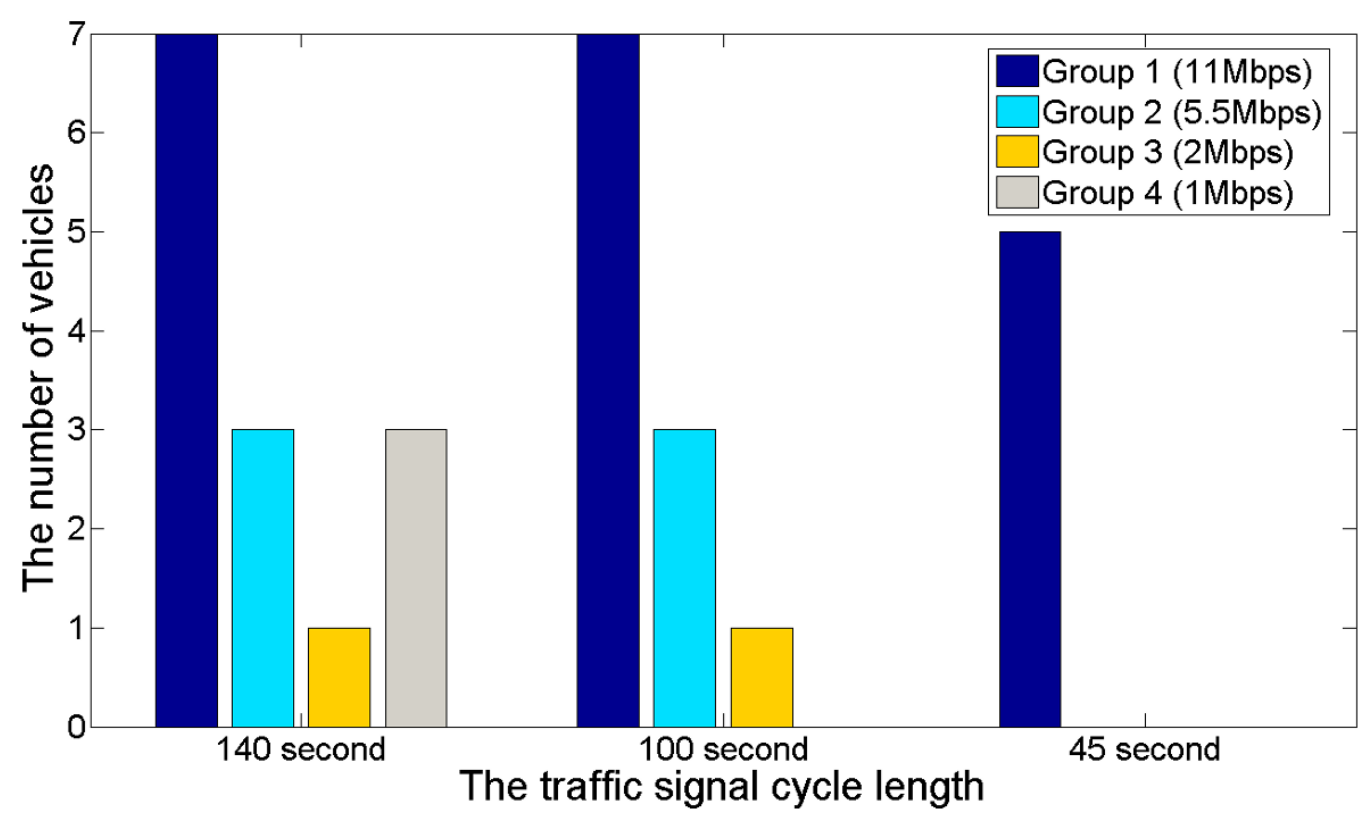

Figure 9 Number of vehicles in each group at one-lane four-way intersection (vehicle arrival rate $=900$ vehicles/hour). 


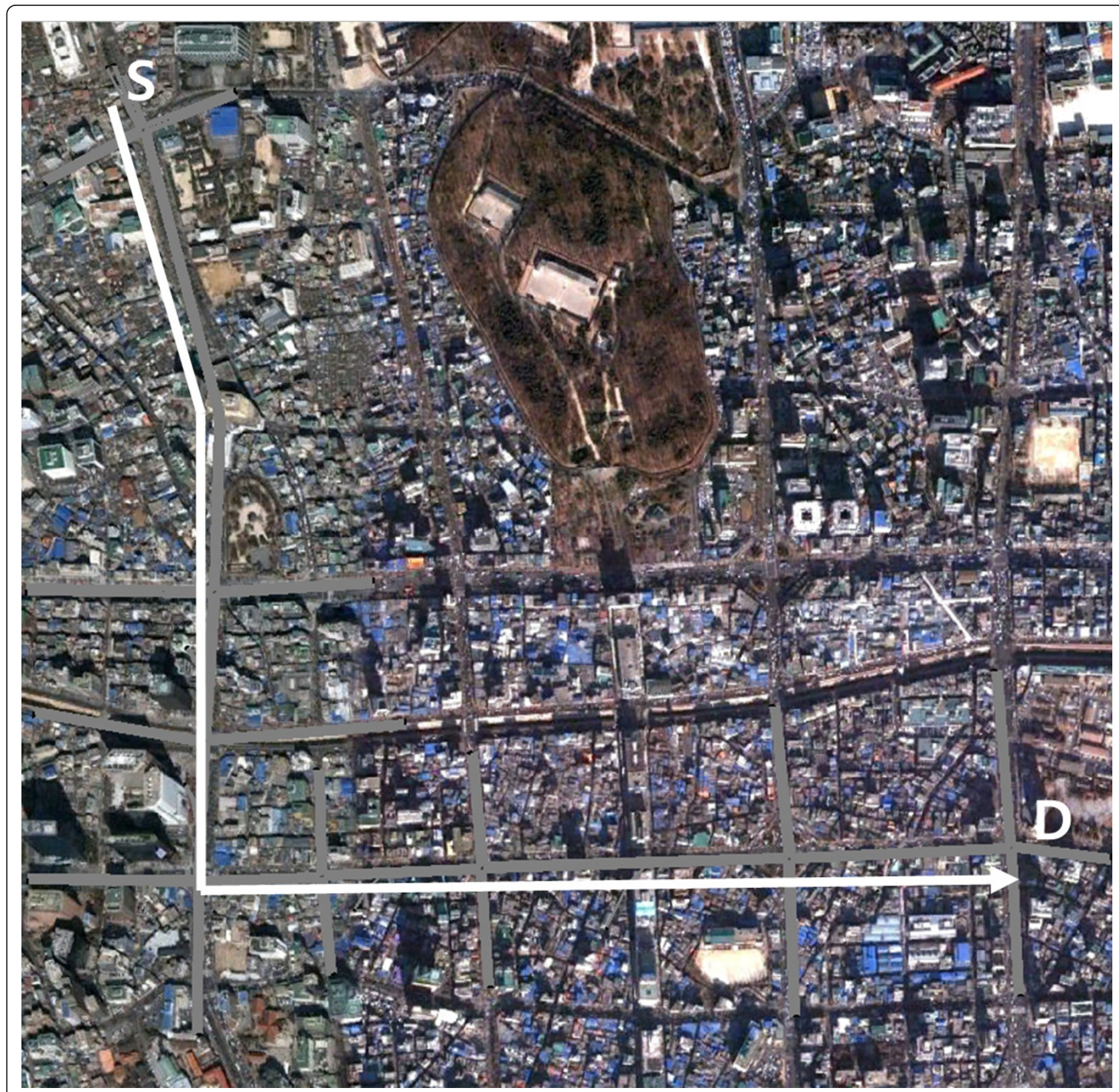

Figure 10 Traveling path at Myeong-dong: Vehicles follow the path from $S$ to $D$.

the super group $(2,3,4)$ cannot transmit any data at some APs and thus starvation issues can occur. However, this starvation issue can be addressed at multiple intersection environments. To justify this, more realistic topologies are considered and the effects of mobility and topology in urban environments are investigated.

In urban environments, vehicles usually move through multiple intersections. Hence, we consider traveling paths including multiple intersections in two urban areas at Korea (i.e., Myeong-dong and Gangnam) as shown in Figures 10 and 11. Vehicles following these paths are observed to measure the average received data and the minimum received data by a vehicle during a travel. We assume that every road segment around intersections has full traffic density, i.e., each lane is fully populated by vehicles up to the end of the transmission range of an AP.

Figures 12 and 13 show the average received data by a vehicle at Myeong-dong and Gangnam, respectively, for different numbers of intersections and for each super group associated with the AP. Intuitively, the total number of bytes increases as the number of intersections increases and/or groups using low transmission rates are excluded. In particular, it can be found that the 


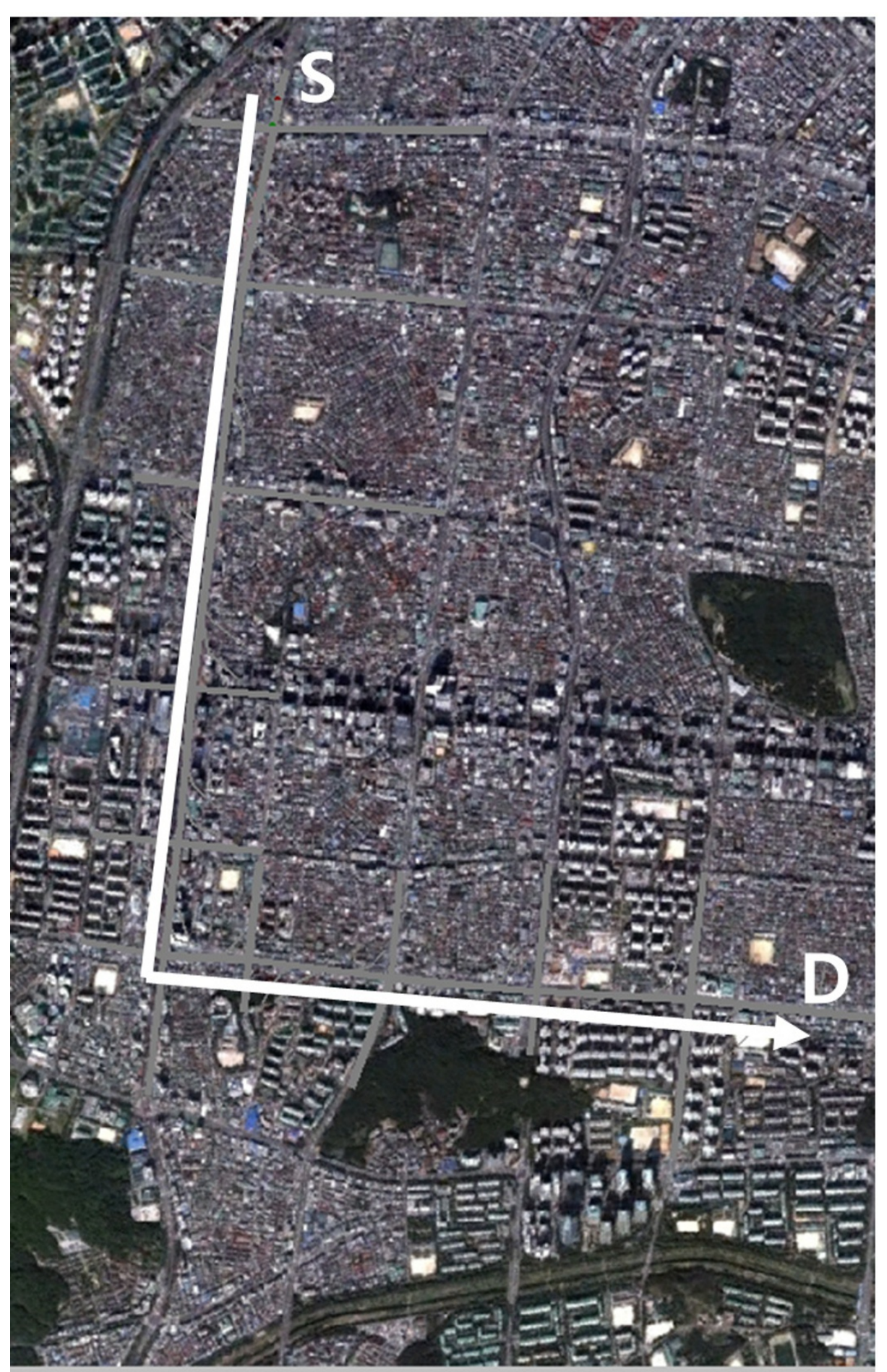

Figure 11 Traveling path at Gangnam: Vehicles follow the path from $S$ to $D$

exclusion of groups using low transmission rates leads to significant performance enhancement compared to the case when the admission control is not used (i.e., super group $(1,2,3,4))$. It is the best choice to support the super group (1) in terms of average throughput regardless of the number of intersections. However, in this case, more vehicles are excluded through admission control and they can experience starvation.

As depicted in Figure 14, in the worst case, some vehicles may not receive any data at all even after passing 


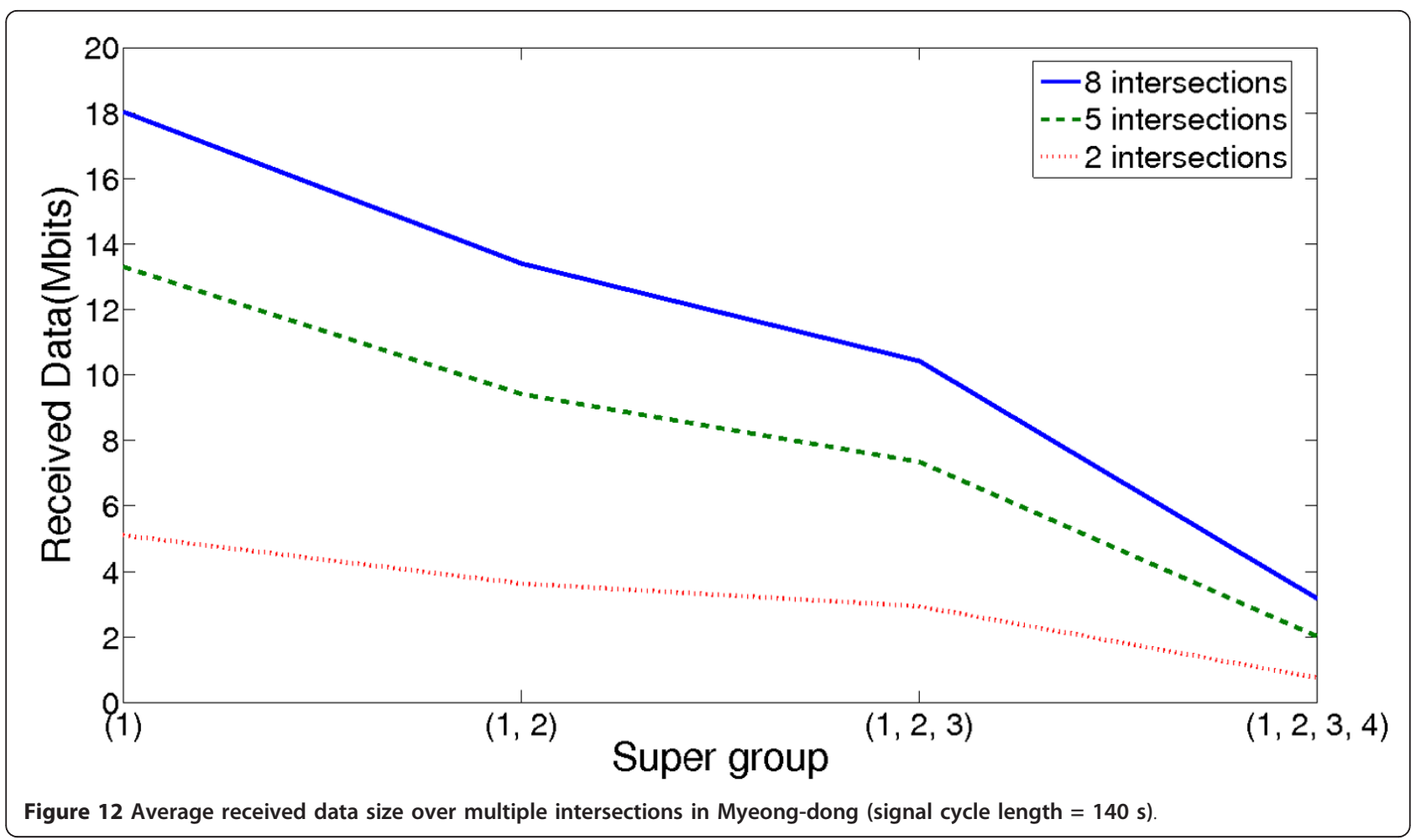

through two intersections when the AP allows only super groups $(1)$ or $(1,2)$. However, as the number of intersections increases, vehicles with admission control (i.e., (1), $(1,2)$, and $(1,2,3))$ can receive much more data than the case when no admission control is used (i.e., $(1,2,3,4))$ even in the worst case. In other words, the starvation problem induced by admission control can be mitigated as the number of intersections increases.

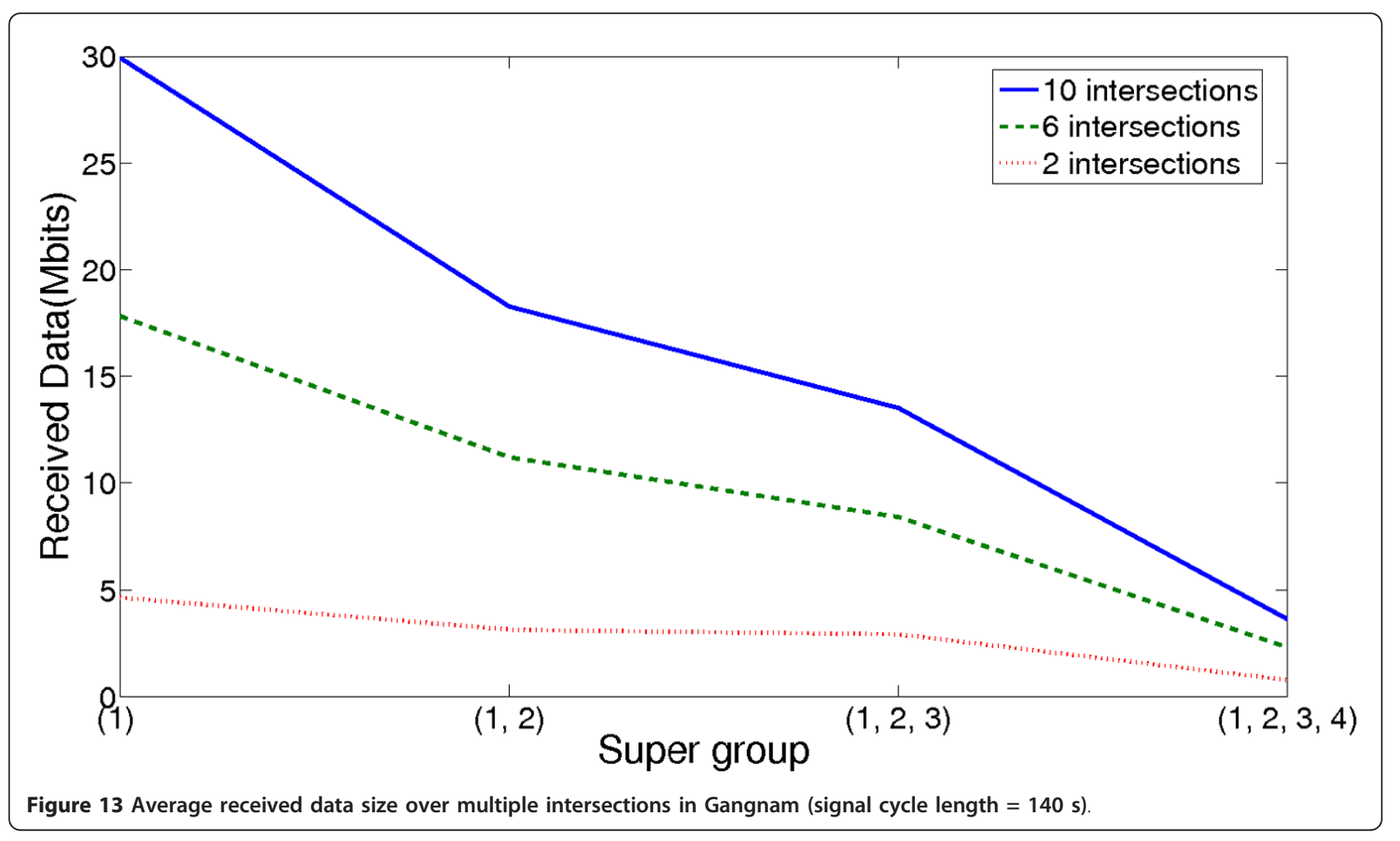




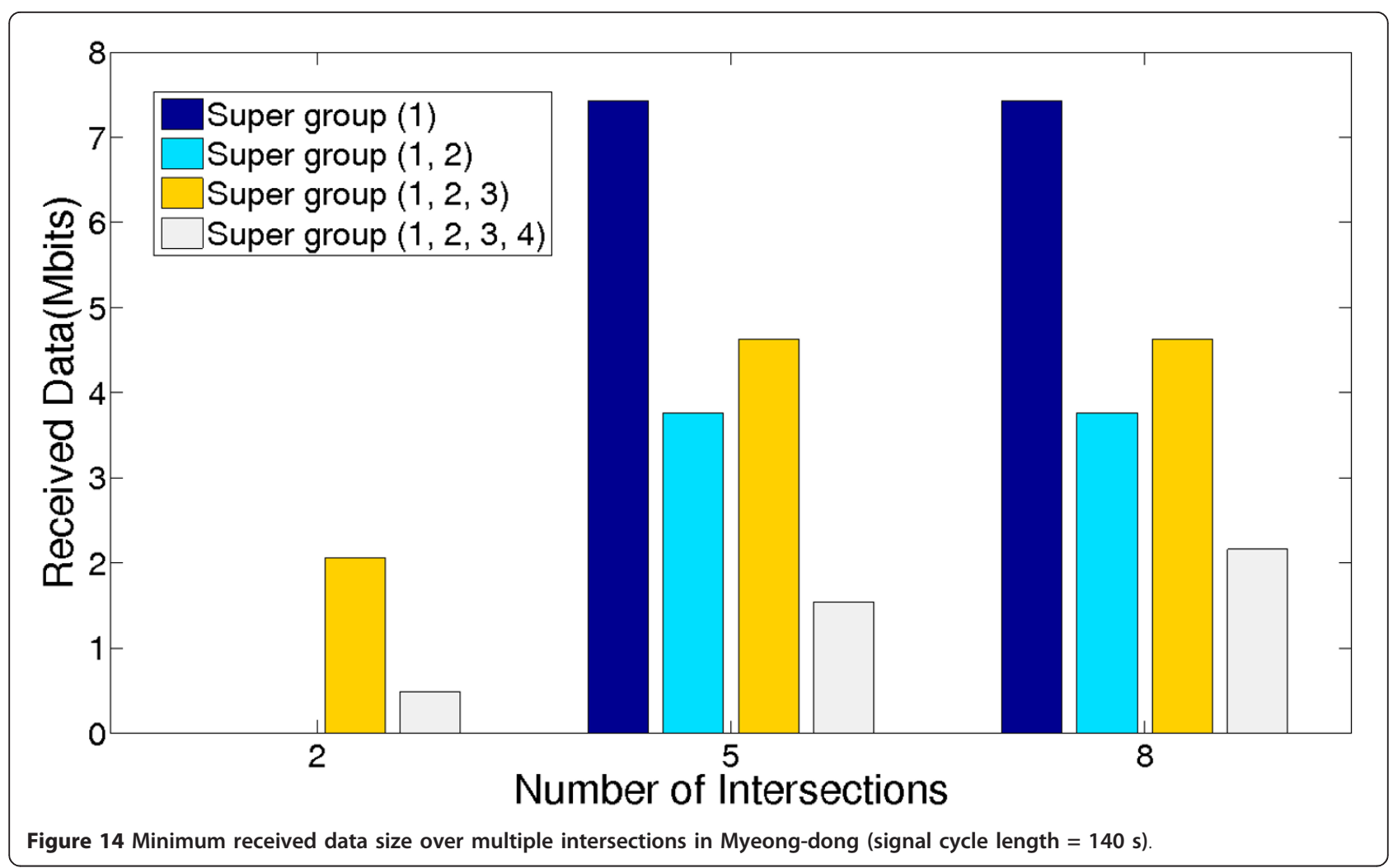

Similar trends can be observed at Gangnam as shown in Figure 15. In short, the starvation issue can be resolved in realistic vehicular environments where a number of intersections exist.

As shown in Figure 8, performance anomaly is apparent for the super group $(1,2,3)$, and therefore we analyze the network throughput of a super group $(1,2,3)$ depending on the number of ways and lanes at a single intersection with the fixed traffic signal cycle length. From Figure 16, it can be seen that throughput degradation occurs earlier at a five-way intersection than others. With the fixed traffic signal cycle length, the red light signal (i.e., the stop signal) length increases with the increase of the number of ways because the traffic signal cycle time is fairly distributed to each road segment. For example, when the signal cycle length is 120,40 , and 30 $\mathrm{s}$ are assigned to each way in three-way and four-way intersections, respectively. Then, a vehicle in the threeway intersection should wait for $80(=40+40)$ s during the red light signal because vehicles in other two lanes should pass away. In the same manner, a vehicle in the four-way intersection should wait for $90(=30+30+$ 30) s during the red light. As a result, for a five-way intersection, vehicles are accumulated during a longer time period and performance anomaly happens earlier if the fixed traffic signal cycle length is assumed.
To see the effect of the number of lanes at an intersection, the network throughput of a super group $(1,2$, 3 ) is analyzed with respect to the vehicle arrival rate at a four-way intersection as shown in Figure 17. As the number of lanes increases, the space available for vehicles at each road segment enlarges and thus more vehicles can be located close to an AP. Hence, vehicles can communicate with an AP at a higher transmission rate and performance anomaly happens slowly as the number of lanes increases.

\section{Conclusion}

In this article, we proposed an SNR-based admission control scheme to address the performance anomaly problem in WiFi-based vehicular networks. Even though a starvation problem can occur at a single intersection $\mathrm{AP}$, its impact becomes insignificant as the number of intersections increases (i.e., multiple intersection environments). Simulation and analytical results show that the SNR-based admission control scheme can improve the network throughput and mitigate the effects of performance anomaly. As future studies, we will devise more sophisticated admission control schemes for IEEE 802.11p networks, in which more accurate channel estimation methods will be introduced and fairness issues will be investigated. 


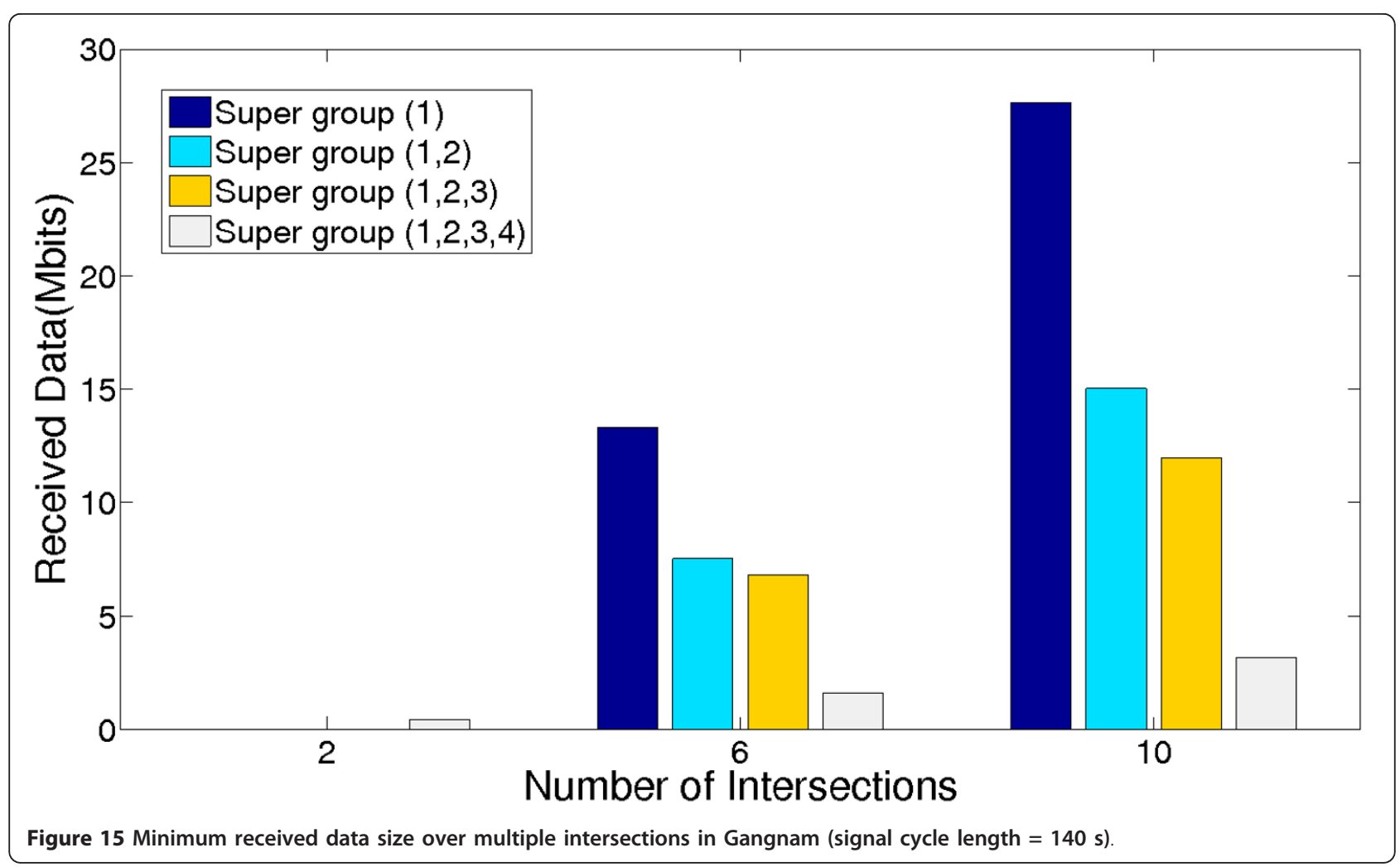

\section{Endnotes}

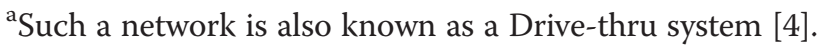
b When a vehicle has high speed, the channel condition at the frame transmission may be different from the channel condition at the association, and thus the admission control result may not be appropriate. However, since the vehicle will pass away the AP quickly, the impact of high speed vehicle is limited to the short time and therefore we do not design any special mechanism for high-speed vehicles. ${ }^{\mathrm{c}}$ In IEEE 802.11p, an amendment for wireless access

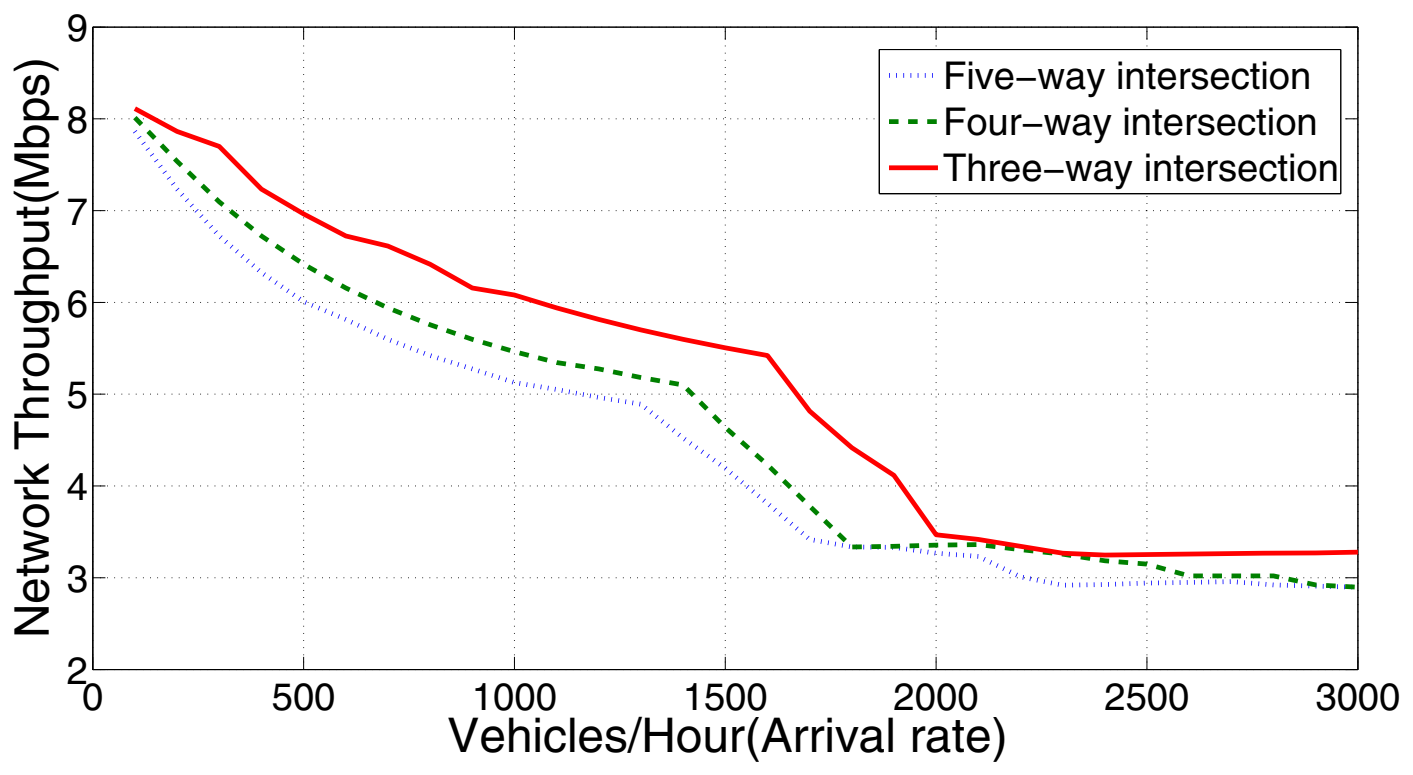

Figure 16 Network throughput versus number of ways at intersection (signal cycle length = $140 \mathrm{~s}$ and super group (1,2,3)) 


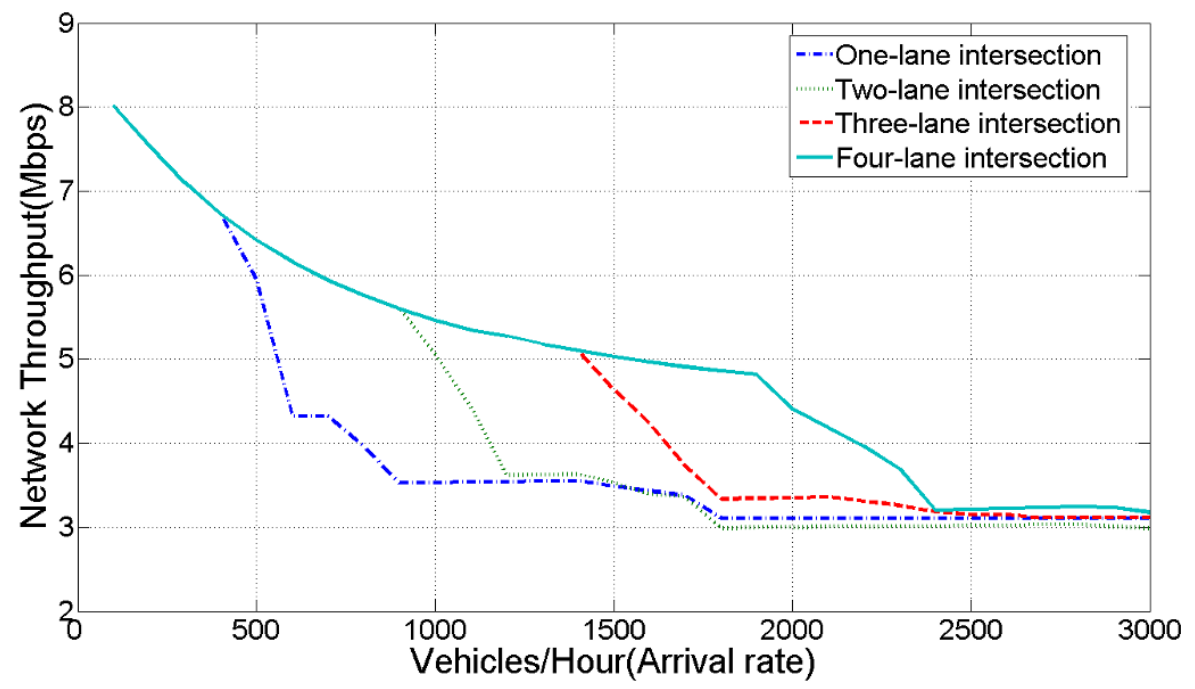

Figure 17 Network throughput versus number of lanes (signal cycle length $=140 \mathrm{~s}$ and super group $(1,2,3)$ ).

in vehicular environments (WAVE), there are no interactive steps between the AP and nodes to perform association operation. However, our work is based on commodity WiFi techniques such as $802.11 \mathrm{~b} / \mathrm{a} / \mathrm{g}$. The extension to IEEE 802.11p is one of our future works.

\section{Acknowledgements}

This study was supported in part by the National Research Foundation of Korea Grant funded by the Korean Government (2009-0064397) and in part by the BLS project funded by Seoul Metropolitan City (Seoul R\&BD Program (WR080951). A preliminary version of this article was presented at International Wireless Communications and Mobile Computing (IWCMC) Conference 2011, July 2011.

\section{Author details}

${ }^{1}$ School of Electrical Engineering, Korea University, Seoul, Korea ${ }^{2}$ AlcatelLucent, Bell-Labs Seoul, Seoul, Korea

\section{Competing interests}

The authors declare that they have no competing interests.

Received: 29 June 2011 Accepted: 19 December 2011 Published: 19 December 2011

\section{References}

1. A Balasubramanian, R Mahajan, A Venkataramani, BN Levine, J Zahorjan, Interactive WiFi connectivity for moving vehicles. Proc ACM SIGCOMM 2008. (2008)

2. S Tenorio, P Spence, B Garriga, J Lopez, A Garcia, M Arranz, 3G HSPA for broadband communications with high speed vehicles. Proc IEEE VTC 2010 (2010)

3. BB Chen, MC Chan, MobTorrent: a framework for mobile internet access from vehicles. Proc IEEE INFOCOM 2009. (2009)

4. J Ott, D Kutscher, Drive-thru internet: IEEE 802.11b for "automobile" users. Proc IEEE INFOCOM 2004. (2004)

5. J Camp, E Knightly, Modulation rate adaptation in urban and vehicular environments: cross-layer implementation and experimental evaluation. Proc ACM MOBICOM 2008. (2008)

6. M Heusse, F Rousseau, G Berger-Sabbatel, A Duda, Performance anomaly of 802.11b. Proc IEEE INFOCOM 2003. (2003)
7. J Dunn, M Neufeld, A Sheth, D Grunwald, J Bennett, A practical cross-layer mechanism for fairness in 802.11 networks. Proc BROADNETS 2004. (2004)

8. M Heusse, F Rousseau, R Guillier, A Duda, Idle sense: an optimal access method for high throughput and fairness in rate diverse wireless LANs. Proc ACM SIGCOMM 2005. (2005)

9. B Sadeghi, V Kanodia, A Sabharwal, E Knightly, Opportunistic media access for multirate ad hoc networks. Proc ACM MOBICOM 2002. (2002)

10. V Bychkovsky, B Hull, A Miu, H Balakrishnan, S Madden, A measurement study of vehicular internet access using in situ Wi-Fi networks. Proc ACM MOBICOM 2006. (2006)

11. Z Zheng, Z Lu, P Sinha, S Kumar, Maximizing the contact opportunity for vehicular internet access. Proc IEEE INFOCOM 2010. (2010)

12. N Banerjee, M Corner, D Towsley, B Levine, Relays, base stations, and meshes: enhancing mobile networks with infrastructure. Proc ACM MOBICOM 2008. (2008)

13. W Tan, W Lau, O Yue, T Hui, Analytical models and performance evaluation of drive-thru internet systems. IEEE J Sel Areas Commun. 29(1):207-222 (2011)

14. J Fricker, R Whitford, Fundamentals of Transportation Engineering: A Multimodal Systems Approach. (Prentice Hall, 2004)

15. A Klar, R Kuehne, R Wegener, Mathematical models for vehicular traffic. Surv Math Indus. 6, 215-239 (1996)

16. T Woensel, N Vandaele, Modeling traffic flows with queueing models: a review. Asia Pac J Oper Res. 24, 435-461 (2007). doi:10.1142/ S0217595907001383

17. A Wiesel, J Goldberg, H Messer-Yaron, SNR estimation in time-varying fading channels. IEEE Trans Commun. 54(5):841-848 (2006)

18. VISSIM.http://WwW.ptvamerica.com/support/vissim

19. VISSIM 5.30 User Manual, 128-145 (2010)

20. P Liu, Z Tao, S Panwar, A cooperative MAC protocol for wireless local area networks. Proc IEEE ICC 2005. (2005)

\section{doi:10.1186/1687-1499-2011-204}

Cite this article as: Kim et al:: An SNR-based admission control scheme in WiFi-based vehicular networks. EURASIP Journal on Wireless Communications and Networking 2011 2011:204. 